Pacific Journal of Mathematics

THE KLEIN GROUP AS AN AUTOMORPHISM GROUP 


\title{
THE KLEIN GROUP AS AN AUTOMORPHISM GROUP WITHOUT FIXED POINT
}

\author{
S. F. BAUMAN
}

An automorphism group $V$ acting on a group $G$ is said to be without fixed points if for any $g \in G, v(g)=g$ for all $v \in V$ implies that $g=1$. The structure of $V$ in this case has been shown to influence the structure of $G$. For example if $V$ is cyclic of order $p$ and $G$ finite then John Thompson has shown that $G$ must be nilpotent. Gorenstein and Herstein have shown that if $V$ is cyclic of order 4 then a finite group $G$ must be solvable of $p$-length 1 for all $p|| G \mid$ and $G$ must possess a nilpotent commutator subgroup.

In this paper we will consider the case where $G$ is finite and $V$ noncyclic of order 4 . Since $V$ is a two group all the orbits of $G$ under $V$ save the identity have order a positive power of 2. Thus $G$ is of odd order and by the work of FeitThompson $G$ is solvable. We will show that $G$ has $p$-lengh 1 for all $p|| G \mid$ and $G$ must possess a nilpotent commutator subgroup.

REMARK. It would be interesting to have a direct proof of solvability without resorting to the work of Feit-Thompson.

From now on in this paper $G$ represents a finite group admitting $V$ as a noncyclic four group without fixed points. If $X$ is a group admitting an automorphism group $A$ then $Z(X), \Phi(X), X-A$ will be respectively the center of $X$, the Frattini subgroup of $X$ and the semi-direct product of $S$ by $A$ in the holomorph of $X$. All other notations are standard.

Suppose $V=\left\{v_{1}, v_{2}, v_{3}\right\}$ where the $v_{i}$ are the nonidentity elements of $V$. Denote by $G_{i}$ the set of elements which are left fixed by $v_{i}$. These are easily seen to be $V$-invariant subgroups of $G$ and by a result of Burnside ([1] p. 90) $G_{i}$ are Abelian and $v_{j}$ restricted to $G_{i}$ is the inverse map if $i \neq j$. These subgroups $G_{i}$ are in a sense the building blocks of $G$.

LEMMA 2. ([4] p. 555)

(i) $|G|=\left|G_{1}\right|\left|G_{2}\right|\left|G_{3}\right|$

(ii) $G=G_{1} G_{2} G_{3}$

(iii) Every element $g \in G$ has a unique decomposition $g=g_{1} g_{2} g_{3}$, $f_{i} \in G_{i}$.

LEMMA 2. If $|G|=h m$ where $(h, m)=1$ then $G$ contains a unique $V$ invariant group $H$ such that $|H|=h$. 
Proof. Since $G$ is solvable by Hall ([5] p. 141) groups of order $h$ exist and are conjugate in $G$. Thus there exist an odd number of them permuted by $V$. Since all the orbits have order power of 2 at least one group say $H$ is $V$ invariant. By Lemma $1 H=H_{1} H_{2} H_{3}$ where clearly $H_{i}=H \cap G_{i}$ and $(G: H)=\left(G_{1}: H_{1}\right)\left(G_{2}: H_{2}\right)\left(G_{3}: H_{3}\right)$. Thus the $H_{i}$ are Hall subgroups of the Abelian $G_{i}$ and thus uniquely determined by $G_{i}$ rather than $H$.

The decomposition of a $V$ invariant group $X$ into $X_{1} X_{2} X_{3}$ will play an important role in what will follow. The $X_{i} \leqq G_{i}$ are always $V$ invariant and it is clear that if $\left|X_{i}\right|=1$ for any $i$ then $X$ is Abelian. For example if $X=X_{1} X_{2}$ is $V$ invariant and normalized by a subgroup $K_{1} \subseteq G_{1}$ then $K_{1} X=K_{1} X_{1} X_{2}$ is Abelian. Thus subgroups of the complex $G_{1} G_{2}$ are centralized by elements in $G_{1} G_{2}$ which normalize them. If $X=X_{1}$ then even a stronger statement is available.

\section{Lemma 3. If $X \subseteq G_{i}$, then $N_{G}(X)=C_{G}(X)$.}

Proof. Suppose $i=1$. It is easy to see that $N=N_{G}(X)$ is $V$ invariant and thus $N=N_{1} N_{2} N_{3}$. By the above remark $X N_{2}$ and $X N_{3}$ are Abelian. Since $X N_{1}$ is Abelian, the result follows.

Before we continue to the main results, we must examine the inheritance properties of groups admitting automorphism groups without fixed points. If $G$ is such a group and $H$ is a $V$ invariant subgroup, then clearly $H$ is also such a group. If $K$ is a normal $V$ invariant subgroup of $G$, there exists the canonical way of inducing $V$ on $G / K$. This definition gives rise to an automorphism group $\bar{V}$ acting on $G / K$.

LEMMA 4. ([4] p. 556) In above situation

(i) $\bar{V}$ is without fixed points on $G / K$.

(ii) $(G / K)_{i}=G_{i} K / K$.

Lemma 5. Suppose $V$ acts on $M$ and $A$ without fixed points. Suppose also that $\Lambda$ is an elementary Abelian p-group where $(p,|M|)=1$ and $M$ is acting faithfully on $\Lambda$. If the complex $M_{1} M_{2}$ is a normal subgroup of $M$ and $\Lambda_{i} \neq\{1\} i=1,2,3$ then $\Lambda$ is $M-V$ reducible.

Proof. By Maschke's theorem it will suffice to show that some proper subgroup of $\Lambda$ is $M-V$ invariant. Now $C=C_{A}\left(M_{1} M_{2}\right)$ is $M-V$ invariant and $C \neq \Lambda$ since $M$ acts faithfully. Hence if $|C| \neq 1$ we are done and so we may assume $|C|=1$. Now $\Lambda_{2} \Lambda_{3}$ is the subset of $\Lambda$ inverted by $v_{1}$ and so is invariant under $M_{1}-V$. Set $K=\cap m_{2}\left(\Lambda_{2} \Lambda_{3}\right)$ where the intersection is taken over all $m_{2} \in M_{2}$. Since $M_{1}-V$ normal- 
izes $M_{2}, K$ is $M_{1} M_{2}-V$ invariant. Furthermore, $\Lambda_{2} \subseteq K$ since $M_{2}$ centralizes $A_{2}$. If $K=\Lambda_{2}$, then $M_{1} M_{2}$ centralizes $K$ so $K \subseteq C=\{1\}$ contrary to the fact that $\left|\Lambda_{2}\right| \neq 1$. Thus we must have that $\left|K \cap A_{3}\right| \neq 1$. But then if $R=\cap m_{3}(K)$ where the intersection is taken over all $m_{3} \in M_{3}$ we have that $\{1\} \cong \Lambda \cap K_{3} \cong R \cong \Lambda_{1} \Lambda_{2} \subset \Lambda$. Since $M_{1} M_{2}$ is normal in $M$ and $M_{3}$ is $V$ invariant it follows that $R$ is $M-V$ invariant and proper in $\Lambda$. This completes the proof of the lemma.

\section{TheORem 1. For all $p|| G \mid G$ has p-length 1.}

Proof. We prove the theorem by induction on $|G|$. We may assume $G$ has no normal $p^{\prime}$-groups and $P_{0} \neq\{1\}$ is the maximal normal $p$-group of $G$. By Hall ([5] p. 332) we have $C_{G}\left(P_{0}\right) \subseteq P_{0}$. By Lemma 2 , the fact that $P_{0}$ is self centralizing and induction, we may assume $G=P Q=Q P$ where $P$ and $Q$ are $V$ invariant $p$ and $q$ Sylow groups of $G$. By induction we also get that $Q P_{0} \triangleleft G,\left(P: P_{0}\right)=p$ and $P_{0}$ is elementary Abelian. By ([2] p. 795) $Q$ possesses a characteristic subgroup $C$ such that class $(C) \leqq 2, C / Z(C)$ is elementary Abelian and the only automorphisms of $G$ which become the identity when restricted to $C$ have order a power of $q . P C$ is then a $V$ invariant group and by induction if $C \neq Q$, since $P_{0}$ is self centralizing we get $P \triangleleft P C$. Thus $P C / P_{0}=P / P_{0} \times C P_{0} / P_{0}$. Since $P / P_{0}$ does not centralize $Q P_{0} / P_{0}$ this contradicts the choice of $C$. Thus $Q=C$. Since $P$ is normal in any proper $V$ invariant subgroup containing it we get that $\left(P / P_{0}-V\right)$ is irreducible on $Q P_{0} / \Phi(Q) P_{0}$. Thus either $Q$ is Abelian or $Z(Q) \subseteq \Phi(Q)$. Since $Q / Z(Q)$ is elementary we get that $Z(Q)=\Phi(Q)$. Thus either $Q$ is Abelian or nonabelian of class 2 with $Z(Q)=\Phi(Q)$. Since $|P| P_{0} \mid=p$ we may suppose $P / P_{0}=\left(P / P_{0}\right)_{3}$. By the irreducibility of $P / P_{0}-V$ on

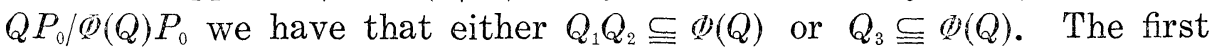
possibility implies that $P / P_{0}$ centralizes $Q P_{0} / \Phi(Q) P_{0}$ and thus $P$ would be normal in $G$. Thus we have that $Q_{3} \subseteq Z(Q)$ and since $Q / Q_{3}$ is Abelian we have $Q_{1} Q_{2} \triangleleft Q$ and $Q_{2} Q_{3} \triangleleft Q$.

Since $Q_{1} Q_{2}$ does not centralize $P_{0}$, there exists an irreducible $Q-V$ submodule $A$ of $P_{0}$ which is not centralized by $Q_{1} Q_{2}$. Thus $\Lambda_{3} \neq\{1\}$. Since $Q P_{0} / P_{0} \triangleleft G_{0} / P_{0}$ we have that $\cap x(\Lambda)$ where $x$ ranges through $P / P_{0}$ is a $G / P_{0}-V$ subspace of $\Lambda$. Since $P / P_{0}=\left(P / P_{0}\right)_{3}$ and $\Lambda_{3} \neq\{1\}$ this space is not the identity space. By the irreducibility of $A$ as a $Q-V$ space we get that $\Lambda$ is also $G / P_{0}-V$ irreducible. If $\Lambda_{i}=\{1\} i=1$ or $i=2$ we get that $\left(P / P_{0}\right)_{3} \subset \operatorname{Ker} \theta$ where $\theta$ maps $G / P_{0}$ into Aut $(\Lambda)$. Since $Q$ does not centralize $A$ this mapping is not the identity and the result follows by induction. Thus $A=\Lambda_{1} \Lambda_{2} \Lambda_{3}$ where $\Lambda_{i} \neq\{1\} i=1,2,3$.

We have that $A$ admits $G / P_{0}$ and thus form the extension $G^{*}=$ $\Lambda \cdot G / P_{0} . \quad G^{*}$ is $V$ invariant and if $\left|G^{*}\right|<|G|$ we may apply induction 
to $G^{*}$. Let $R / P_{0}$ be the maximal normal $q$-subgroup of $G^{*}$. Since $Q$ does not centralize $A$ we have that $R / P_{0}$ is a proper $V$ invariant subgroup of $Q P_{0} / P_{0}$. Since $G^{*}$ has $p$-length $1, \Lambda\left(P R / P_{0}\right) \triangleleft G^{*}$. Thus $P R / P_{0} \triangleleft G / P_{0}$ and $P R \neq G$. We are done by induction on $P R$. We may assume that $\Lambda=P_{0}$. But since $\Lambda_{i} \neq\{1\} i=1,2,3$ and $Q-B$ is faithful irreducible on $P_{0}$ we have a contradiction to Lemma 5. This completes the proof of Theorem 1.

THEOREM 2. If $G$ admits $V$ without fixed points then $G^{\prime}=(G, G)$ is nilpotent.

Proof. Suppose $G$ contains two distinct minimal normal $V$ invariant subgroups $N_{1}$ and $N_{2}$. If $N_{1}$ is disjoint from $G^{\prime}$ tden by induction on $G / N_{1}$ the theorem is proved. If $N_{1}$ and $N_{2}$ are in $G^{\prime}$ then by induction $G^{\prime} / N_{1}$ and $G^{\prime} / N_{2}$ are nilpotent. The minimality of $N_{i}$ imply that the mapping of $G^{\prime}$ into $G^{\prime} / N_{1} \times G^{\prime} / N_{2}$ is an imbedding and thus again we are done. Therefore $G$ contains a unique minimal normal $V$ invariant group. It is an elementary Abelian $p$-group $P_{0}$ which is characteristic. $G$ must contain no normal $p^{\prime}$-groups and by Theorem 1 we have that $G$ has a normal $p$-Sylow group $P$. Now $C_{G}(P)=Z(P) \times K$ where $K$ is a characteristic therefore $V$ invariant $p^{\prime}$-group of $C_{G}(P)$. Since $C_{G}(P) G$ we get that $C_{G}(P)=Z(P) \subseteq P$. Consider $G / \Phi(P)$. If induction applies $G^{\prime} \Phi(P) / \Phi(P)$ is a nilpotent group and since $C_{G / \Phi(P)}(P / \Phi(P))=P / \Phi(P)$ we must have that $G^{\prime} \Phi(P) / \Phi(P)$ is a $p$-group and therefore so is $G^{\prime}$. Thus we have that $P$ is elementary Abelian. Let $M$ be a $V$ invariant complement to $P$ in $G$. By Maschke's theorem and the remark on the number of minimal $V$ invariant normal subgroups of $G$ we have that $P=P_{0}$ and $P$ is $(M-V)$ irreducible.

Consider any proper $V$ invariant subgroup $K$ of $M$. Then $P K \subset G$. By induction $P K$ has a nilpotent commutator subgroup. Since $C_{P K}(P)=P$ this must be a $p$-group and therefore contained in $P$. Since $P K / P \cong K$ we must have that $K$ is Abelian. Thus every proper $V$ invariant subgroup of $M$ is Abelian. If $M$ is Abelian then $G^{\prime} \subseteq P$ and we are done. We assume henceforth that $M$ is not Abelian. Thus $M=M_{1} M_{2} M_{3}$ where $M_{i} \neq\{1\}$ for any $i$. Since $C_{G}(P)=P, P=$ $P_{1} P_{2} P_{3}$ where $P_{i} \neq\{1\}$ for any $i$.

If $M$ contains two $V$ invariant subgroups $K$ and $L$ of prime index, then since these are both Abelian we get that some $M_{i}$ say $M_{1} \subset Z(M)$. Thus $M_{1} M_{2}$ and $M_{1} M_{3}$ are normal in $M . \quad M-V$ is faithful and irreducible on $P$. This situation is in contradiction to Lemma 5. Since $M$ is solvable and $V$ invariant, we have a $V$ invariant Sylow system. If more than two primes divide $|M|$ then we would have $M$ Abelian. If $M$ is a $q$-group for some prime $q$, we can get an $M_{i} M_{j} M$. Thus to avoid this case we are forced to the following situation. $R$ and $S$ 
are $V$ invariant $r$ and $s$ Sylow subgroups, each is Abelian and $M=$ $R S=S R$. We may suppose that $M$ contains a $V$ invariant normal Abelian subgroup $K$ such that $(M: K)=s$. Thus $R M$ and $S$ is cyclic. Thus $S \subseteq M_{i}$ for some $i$. To be specific suppose $S \cong M_{1}$. Then by Maschke's theorem applied to $S_{1}$ acting on $R_{1} R_{2} R_{3} / \Phi(R)$ we get that $R_{2} R_{3}=M_{2} M_{3}$ is normalized by $S_{1}$ and thus $M_{2} M_{3} M$. We have $(M-V)$ irreducible and faithful on $P=P_{1} P_{2} P_{3}$, and we again contradict Lemma 5 . This completes the proof of Theorem 2 .

\section{BIBLIOGRAPHY}

1. W. Burnside, Theory of Groups of Finite Order, Cambridge University Press, 2nd Edition, 1911.

2. W. Feit and J. Thompson, Solvability of groups of odd order, Pacific J. Math. 13 (1963), 775-1029.

3. D. Gorenstein and I. Herstein, Finite groups admitting a fixed point free automorphism of order 4, Amer J. Math. 83 (1961), 71-78.

4. D. Gorenstein and J. H. Walter, On finite groups with dihedral sylow 2-subgroups, Illinois J. Math. 6 (1962), 553-593.

5. M. Hall, The theory of groups, Macmillan, 1959.

6. J. Thompson, Finite groups with fixed point free automorphisms of prime order, Proc. Nat. Acad. Sci. 45 (1959), 578-581.

Received March 3, 1965. This work is contained essentially in the author's doctoral dissertation at the Univ. of Illinois, June 1962. 



\section{PACIFIC JOURNAL OF MATHEMATICS}

H. SAMELSON

Stanford University Stanford, California

R. M. BLUMENTHAL

University of Washington

Seattle, Washington 98105

\section{EDITORS}

\author{
*J. DugundJI \\ University of Southern California \\ Los Angeles, California 90007 \\ RICHARD ARENS \\ University of California \\ Los Angeles, California 90024
}

\section{ASSOCIATE EDITORS}

E. F. BeCKENBACH

B. H. NEUMANN

F. WOLF

K. YosidA

\section{SUPPORTING INSTITUTIONS}

UNIVERSITY OF BRITISH COLUMBIA

CALIFORNIA INSTITUTE OF TECHNOLOGY

UNIVERSITY OF CALIFORNIA

MONTANA STATE UNIVERSITY

UNIVERSITY OF NEVADA

NEW MEXICO STATE UNIVERSITY

OREGON STATE UNIVERSITY

UNIVERSITY OF OREGON

OSAKA UNIVERSITY

UNIVERSITY OF SOUTHERN CALIFORNIA
STANFORD UNIVERSITY

UNIVERSITY OF TOKYO

UNIVERSITY OF UTAH

WASHINGTON STATE UNIVERSITY

UNIVERSITY OF WASHINGTON

AMERICAN MATHEMATICAL SOCIETY CHEVRON RESEARCH CORPORATION TRW SYSTEMS

NAVAL ORDNANCE TEST STATION 


\section{Pacific Journal of Mathematics}

Vol. 18, No. 1

March, 1966

Edward Joseph Barbeau, Semi-algebras that are lower semi-lattices ...... 1

Steven Fredrick Bauman, The Klein group as an automorphism group

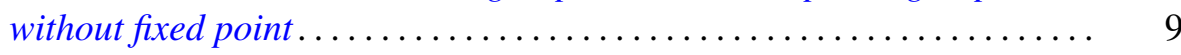

Homer Franklin Bechtell, Jr., Frattini subgroups and $\Phi$-central groups .... 15

Edward Kenneth Blum, A convergent gradient procedure in prehilbert

spaces ............................................

Edward Martin Bolger, The sum of two independent exponential-type random variables ...................................

David Wilson Bressler and A. P. Morse, Images of measurable sets .......

Dennison Robert Brown and J. G. LaTorre, A characterization of uniquely

divisible commutative semigroups........................

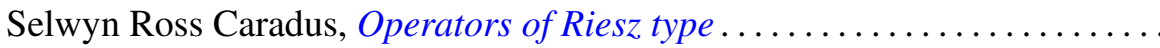

Jeffrey Davis and Isidore Isaac Hirschman, Jr., Toeplitz forms and ultraspherical polynomials ............................

Lorraine L. Foster, On the characteristic roots of the product of certain rational integral matrices of order two ......................

Alfred Gray and S. M. Shah, Asymptotic values of a holomorphic function

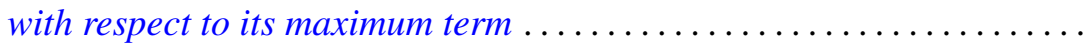

Sidney (Denny) L. Gulick, Commutativity and ideals in the biduals of

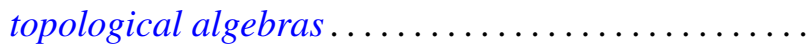

G. J. Kurowski, Further results in the theory of monodiffric functions

Lawrence S. Levy, Commutative rings whose homomorphic images are self-injective .

Calvin T. Long, On real numbers having normality of order $k$....

Bertram Mond, An inequality for operators in a Hilbert space. ...

John William Neuberger, The lack of self-adjointness in three-point boundary value problems ........................

C. A. Persinger, Subsets of $n$-books in $E^{3}$

Oscar S. Rothaus and John Griggs Thompson, A combinatorial problem in the symmetric group ............................... 175

Rodolfo DeSapio, Unknotting spheres via Smale .................. 179

James E. Shockley, On the functional equation

$$
F(m n) F((m, n))=F(m) F(n) f((m, n)) \ldots \ldots \ldots
$$

Kenneth Edward Whipple, Cauchy sequences in Moore spaces ... 